\title{
PERTURBATION OF THE DIRECTION OF NEURITE GROWTH BY PULSED AND FOCAL ELECTRIC FIELDS ${ }^{1}$
}

\author{
NILESH B. PATEL AND MU-MING POO ${ }^{2}$
}

Department of Physiology and Biophysics, University of California, Irvine, California 92717

Received October 20, 1983; Revised May 4, 1984; Accepted May 30, 1984

\begin{abstract}
We have studied the orientation of neurite growth in the culture of embryonic Xenopus neurons in response to three types of extracellular electric fields: spatially uniform pulsed fields, focally applied steady (DC) fields, and focally applied pulsed fields. Under uniform pulsed fields, neurites showed a preferential orientation toward the cathode pole of the field in a manner similar to that previously found for DC fields. The extent of neurite orientation depended upon the duration, amplitude, and frequency of the pulse but appeared to be similar to that produced by a uniform DC field of an equivalent time-averaged field intensity. For square pulses of $5 \mathrm{msec}$ duration, the minimal amplitude and frequency required to produce a detectable orientation of neurite growth over a period of $24 \mathrm{hr}$ were $2.5 \mathrm{~V} / \mathrm{cm}$ and $10 \mathrm{~Hz}$, which correspond to a time-averaged field intensity of 125 $\mathrm{mV} / \mathrm{cm}$. Steady or pulsed focal fields were applied by passing a current through a micropipette placed near the growth cone of the neurite. Fields of negative polarity (current sink) were found to attract the growth cone, whereas fields of positive polarity (current source) were found to deflect the growth cone away from the pipette. The threshold DC current density needed at the growth cone to perturb its direction of growth within 15 min was 0.2 to $2 \mathrm{pA} / \mu \mathrm{m}^{2}$ (or 3 to $30 \mathrm{mV} / \mathrm{cm}$ ); and for focal pulsed currents (pulse duration $5 \mathrm{msec}$ ), a typical combination of minimal pulse amplitude and frequency was $4 \mathrm{pA} / \mu \mathrm{m}^{2}$ and $10 \mathrm{~Hz}$. This threshold focal current is similar to that which occurs at the synaptic cleft during active synaptic activity.
\end{abstract}

Steady (DC) extracellular electric fields have been shown to accelerate and orient the growth of embryonic neurites in cultures of explanted nervous tissue (Marsh and Beams, 1946; Sisken and Smith, 1975; Jaffe and Poo, 1979) and in cultures of dissociated neurons (Hinkle et al., 1981; Freeman et al., 1981; Patel and Poo, 1982). An immediate question that arises is whether these in vitro field effects bear any physiological relevance to neurite growth in vivo. Electric fields generated within the nervous tissue, e.g., those associated with action potentials and synaptic activity, are pulsatile in nature, and because of the nonuniform distribution of current sources and sinks, they will appear as nonuniform and focal electric fields. In the present study, we have examined the response of isolated neurites to the presence of uniformly applied pulsed fields and focally applied DC and pulsed fields, and we have determined the amplitude and frequency required of such fields to perturb the direction of neurite growth. The results provide a basis for future studies on the effects of endogenous fields within the nervous system.

\section{Materials and Methods}

\section{Culture preparation and application of the electric fields}

Preparation of the culture of dissociated embryonic Xenopus neurons was identical to that reported previously (Patel and Poo, 1982). The

\footnotetext{
${ }^{1}$ This work was supported by Grant NS-17558 from the National Institutes of Health. We thank Drs. I. Chow and S. H. Young for helpful comments on the manuscript.

${ }^{2}$ To whom correspondence should be addressed.
}

culture consisted of a heterogeneous population of neurons obtained from the neural tube of the 1-day-old Xenopus embryo (stage 17-19; Nieuwkoop and Faber, 1962). All isolated neurons whose cell bodies and neurites did not contact other cells were used for analysis. All of the experiments were carried out within $24 \mathrm{hr}$ after the cells were plated in the culture chamber, since the average length of the neuritic processes during this period was less than $150 \mu \mathrm{m}$ and a substantial fraction of the neurons remained isolated from other cells in the culture. Moreover, many neurites ceased growth activity after $24 \mathrm{hr}$ in this culture. The culture medium was Steinberg's saline (composition in millimolar concentration): $\mathrm{NaCl}, 58 ; \mathrm{KCl}, 0.7 ; \mathrm{Ca}\left(\mathrm{NO}_{3}\right)_{2}, 0.4 ; \mathrm{MgSO}_{4}$, 0.1 , and Tris, 4.6 , supplemented with $1 \%$ fetal calf serum (Grand Island Biological Co.). The $\mathrm{pH}$ of the culture medium was 7.8 . This simple "hypotonic" medium supports well the survival of these embryonic neurons and was used during the course of all experiments. Both the culture incubation and all of the experimental procedures were carried out at room temperature $\left(21 \pm 1^{\circ} \mathrm{C}\right)$.

Three types of electric fields were used in the present study (in the sequence shown): uniform pulsed field, focal DC field, and focal pulsed field. For each type of study, only one field property was altered. This allows a direct comparison between the results obtained.

Uniform pulsed fields were applied in a rectangular culture chamber identical to that described previously (Patel and Poo, 1982). Monopolar, square current pulses of different pulse duration and frequency were supplied to the culture by a Grass stimulator (SD9) through a pair of $\mathrm{Ag}-\mathrm{AgCl}$ electrodes in two saline reservoirs. The latter were connected to the culture chamber by a pair of agar bridges made with culture medium. The purpose of the agar bridge was to prevent contamination of the culture by electrode products or alteration of the culture medium $\mathrm{pH}$ during the course of the experiment (duration up to $24 \mathrm{hr}$ ). The field strength $(E)$ was estimated from the total current flow $(I)$ through the culture chamber, the cross-sectional area $(A)$ of the chamber, and the resistivity $(\rho)$ of the medium by the formula $E=I \rho / A$. 
Focal fields, either DC or pulsed, were applied directly to the growth cones by passing a current through glass micropipettes filled with $5 \%$ low temperature agarose (Miles Laboratories, Inc.) gelled with culture medium. The tip opening of the pipette was in the range of 3 to $7 \mu \mathrm{m}$ and the electrical resistance was $4.2 \pm 0.7$ megohms $(n=9, \pm \mathrm{SEM})$. The pipettes were positioned at an angle of $45^{\circ}$ with respect to the culture substratum with which the tip of the pipette was in contact. Currents (DC or square pulses) were supplied by a Grass stimulator (SD9) and monitored continuously with a current-to-voltage amplifier through a reference (virtual ground) electrode placed ncar the edge of the circular culture chamber (diameter $=60 \mathrm{~mm}$ ). Assuming that the plastic culture chamber is a perfect insulator and the focal pipette provides a point source (or sink) of current on the surface of an insulator, it can be shown that the current density $(J)$ at a growth cone located a distance $r \mu \mathrm{m}$ from the pipette tip, in the first-order approximation, is given by:

$$
J=I / 2 \pi r^{2}
$$

where $I$ is the total current flow through the point current source. For a 10 -nA current, the current density $30 \mu \mathrm{m}$ away from the pipette tip is $1.8 \mathrm{pA} / \mu \mathrm{m}^{2}$, which corresponds to a field strength of $29 \mathrm{mV} / \mathrm{cm}$ for the culture medium $(\rho=160 \mathrm{ohm}-\mathrm{cm})$ used in this study. Since this formula was derived by assuming the pipette tip to be infinitesmally small, while the actual size of the tip opening was 3 to $7 \mu \mathrm{m}$, the formula was used only for large $r(>15 \mu \mathrm{m})$.

In preliminary studies, we found that there was a substantial bulk flow of medium into or out of the pipettes which were not filled with the agarose gel. This medium flow by itself (in the absence of an electrical current) frequently perturbed the orientation of the growth cone. Therefore, all of the focal field experiments reported here were carried out with agarose-filled pipettes, and the absence of medium flow through the pipettes, when no current was applied, was confirmed by examining the motion of tiny suspended culture debris at the tip opening before and during the course of each experiment.

\section{Analysis of neurite growth and orientation}

Uniform pulsed field. Analysis of the average neurite length, the neurte growth asymmetry, and the asymmetry of neurite initiation on cell soma was identical to that reported previously (Patel and Poo, 1982 ). Briefly, cells were fixed with $2 \%$ glutaraldehyde immediately after the termination of the field. Line drawings of the isolated neurons and their neurite processes were made with the aid of a camera lucida, and the information about the neurite growth was then stored in a microcomputer (Cromenco Z-2D) by using a digitizing pad (Houston Instrument). Using the computer, three indices were calculated to characterize the growth and orientation of the neurite processes: (1) average neurite length (ANL), which was obtained by dividing the sum of the entire trajectory of each neurite process, including the branches, by the total number of neurites traced; (2) neurite growth asymmetry (NGA), which was the normalized difference between the average projected growth of neurites along the field axis toward the cathode and the anode of the field; and (3) neurite initiation site asymmetry (NISA), which was the average cosine value of the neurite outgrowth angle at the soma with respect to the field axis. ANI has units of length (micrometers). Both NGA and NISA are dimensionless, with maximal value of 1 and minimal value of 0 . All three indices were calculated for each culture, and the indices from identically treated cultures were grouped to calculate the average index. The control cultures (not treated with the field) had NGA and NISA within the range of \pm 0.05 . Thus, indices with absolute value larger than 0.05 were considered to represent a significant asymmetry.

Focal field. The response of growth cones to focal electric fields was studied by monitoring the growth and orientation of the growth cone by video recording, using a video camera (RCA) coupled to a video recorder (Panasonic) and a monitor (Hitachi). Analysis of the neurite growth was made by tracing the recorded images directly from the video monitor. At the start of the experiment, the tip of the currentsupply pipette was positioned at a distance of $30 \mu \mathrm{m}$ from the growth cone and at an angle of $45^{\circ}$ with respect to the direction of neurite growth (see Fig. 5a). After the field application, the length of the neurite growth and the angle of neurite orientation were measured for each neurite by comparing the tracings of the growth cone before and after the field. The position of the growth cone was defined as the center of the phase-dark "palm" of the growth cone. The direction of growth was defined as the line that passed through the last $10 \mu \mathrm{m}$ of the neurite tip. An orientation angle, defined as the angle between the growth direction before and after the field application, was used to characterize the turning of the growth cone toward or away from the focal current pipette. A neurite was considered to exhibit a bending response if its direction of growth deviated from the original direction by more than $5^{\circ}$. An orientation index (O.I) was used to determine whether there was any preferential orientation in a population of growth cones. O.I. $=\left(N_{+}-N_{-}\right) / N_{0}$, where $N_{+}$and $N_{-}$are number of growth cones showing bending toward and away from the focal current pipette, respectively, and $N_{0}$ is the total number of growth cones examined, including those that did not respond to the applied current. In control cultures, when no current was applied the absolute value of O.I. was always below 0.2 ; thus absolute O.I. values above 0.2 were considered to represent a significant orientation effect.

\section{Results}

\section{Neurite growth and orientation in pulsed uniform fields}

Dependence on the pulsed field amplitude. In the first set of experiments, we applied monopolar square current pulses of 5 msec duration and pulse amplitudes that gave 1 to $10 \mathrm{~V} / \mathrm{cm}$ fields to a group of freshly plated cultures for $4.5 \mathrm{hr}$ at a frequency of $100 \mathrm{~Hz}$. The growth, orientation, and the initiation sites of the neurites were measured after the field was terminated and the cells were fixed. The average length of neurite growth was not significantly different from that of the control cultures (not exposed to the field): for example, it was $78.8 \pm$ $4.3 \mu \mathrm{m}$ (SEM, 21 cultures) for the control culture and $77.0 \pm$ $2.8 \mu \mathrm{m}$ ( 4 cultures) for the culture exposed to a $5 \mathrm{~V} / \mathrm{cm}$ pulsed field. However, the projected neurite growth (see "Materials and Methods") toward the cathode was increased by the field: $49.7 \pm 1.6 \mu \mathrm{m}$ in the control cultures, and $71 \pm 1.2 \mu \mathrm{m}$ in the cultures treated with a $5 \mathrm{~V} / \mathrm{cm}$ pulsed field; whereas the projected growth toward the anode was reduced by the same pulsed field: $53.4 \pm 2.6 \mu \mathrm{m}$ in the control, and $37.5 \pm 6.1 \mu \mathrm{m}$ in the field. The resultant asymmetry indices (NGA and NISA) from these experiments are depicted in Figure 1. A clear orientation of neurite growth (Fig. 1A) and polarization of neurite initiation site (Fig. $1 B$ ) toward the cathode was observed at pulse field amplitudes above $1.25 \mathrm{~V} / \mathrm{cm}$.

In parallel cultures, we also examined the effects of steady fields of the same time-averaged intensity as the pulsed fields. The resultant asymmetries in the neurite growth and in the neurite initiation were similar for both types of field (see Fig. 1), suggesting that for the pulsed fields of this high duty cycle $(50 \%)$, the time-averaged field intensity may play a dominant role in determining the orientation effect. This notion was further tested by treating cultures with fields of different combinations of pulse amplitudes and frequencies but having the same time-averaged intensity. Two average intensities were tested: 0.25 and $0.675 \mathrm{~V} / \mathrm{cm}$, each at three different sets of pulse amplitude and frequency. The result is shown as histoyrams in Figure 2. For the high average field intensity of 0.675 $\mathrm{V} / \mathrm{cm}$, the three sets of pulsed fields produced nearly identical high asymmetry in both neurite growth and initiation. For the low field strength of $0.25 \mathrm{~V} / \mathrm{cm}$, the combination with the high pulse amplitude $(12.5 \mathrm{~V} / \mathrm{cm}, 4 \mathrm{~Hz})$ appeared to produce a slightly higher neurite growth asymmetry. This asymmetry was significantly different ( $p<0.05, t$ test) from that of $2.5 \mathrm{~V} / \mathrm{cm}$ and $20 \mathrm{~Hz}$, but not significantly different ( $p<0.5, t$ test) from that of $0.5 \mathrm{~V} / \mathrm{cm}$ and $100 \mathrm{~Hz}$. We thus conclude from these experiments that, at least for fields with pulse amplitudes below $10 \mathrm{~V} / \mathrm{cm}$, the time-averaged field intensity appears to be the determining factor for the extent of neurite growth asymmetry.

Pulsed field and DC field of equivalent time-averaged intensity do differ, however, in their effect on the absolute growth rate of neurite: no effect on the ANL was found over the range of pulsed fields examined in the present study, whereas for the DC field of $5 \mathrm{~V} / \mathrm{cm}$, significant increase in the ANL was 

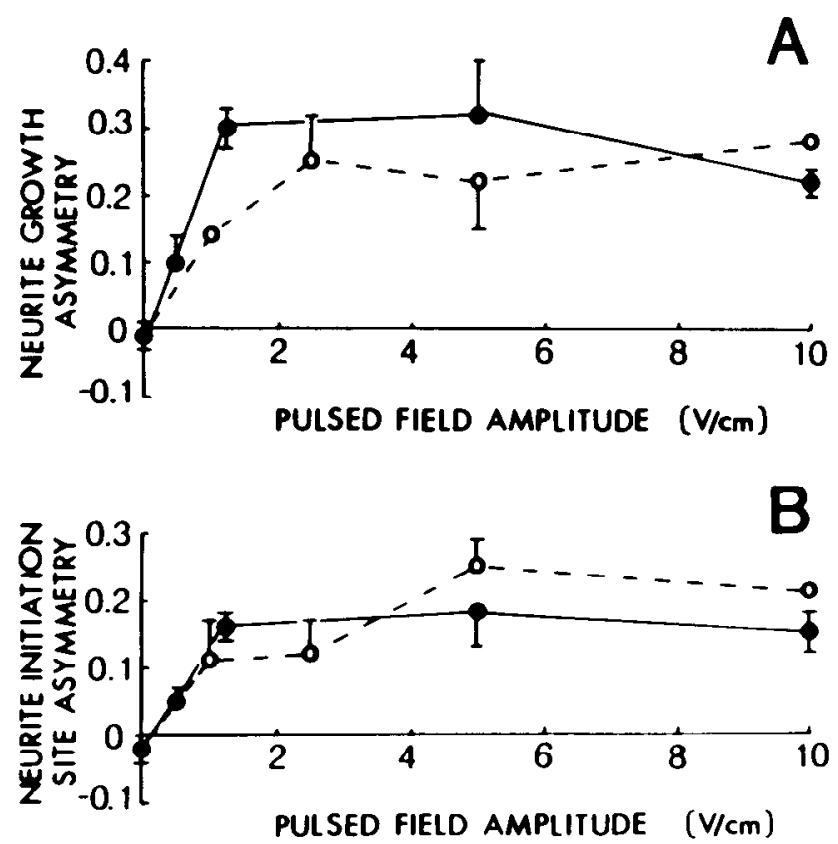

Figure 1. Neurite growth in the presence of pulsed and steady electric fields. Asymmetry indices depict the orientation of the neurite growth (A) and the polarization of the neurite initiation site on the cell soma $(B)$ induced by spatially uniform but monopolarly pulsed fields (solid circles) and by uniform steady (DC) field of the same time-averaged intensity (open circles). The Xenopus neuronal cultures were exposed to a square monopolar pulsed field of $100 \mathrm{~Hz}$ frequency with a 5 -msec pulse duration and different pulse amplitudes for $4.5 \mathrm{hr}$. Asymmetry indices were determined by the procedure discussed under "Materials and Methods." The amplitude of the DC field used was equal to the time-averaged intensity of the corresponding pulsed field; e.g., a DC field of $2.5 \mathrm{~V} / \mathrm{cm}$ was used for the pulsed field of $5 \mathrm{~V} / \mathrm{cm}$ amplitude. Significant orientation in the neurite growth and polarization of the neurite initiation toward the cathode was observed for pulse amplitudes above $1.25 \mathrm{~V} / \mathrm{cm}$. The pulsed field was as effective as the equivalent DC field in inducing asymmetry. Each point represents the average of at least three separate cultures. The error bars represent the standard error of the mean.

observed. The growth-promoting effect of the DC field has been studied previously in detail (Patel and Poo, 1982).

Dependence on the pulse frequency. For square pulses of the same duration and amplitude, the effect on neurite growth direction was frequency dependent. This was shown by experiments in which the frequency of the pulsed fields (pulse duration $5 \mathrm{msec}$ and amplitude of $5 \mathrm{~V} / \mathrm{cm}$ ) was varied from 1 to $100 \mathrm{~Hz}$. These fields were applied for either 4.5 or $24 \mathrm{hr}$. The lowest frequency that produced a detectable asymmetry in the growth orientation was about $25 \mathrm{~Hz}$ and $10 \mathrm{~Hz}$ for 4.5 - and 24 hr treatments, respectively (Fig. 3 ). This frequency dependence is consistent with the notion that the field effect is determined by the time-averaged field intensity: lower frequency field yields lower time-averged intensity and thus produces smaller effects. Comparing the effects due to the same frequency at 4.5 and 24 $\mathrm{hr}$, we found that there was no significant difference in the NISA, but a much larger NGA was observed for the 24-hr treatment at $50 \mathrm{~Hz}$. This suggests that the field effect on the neurite initiation site was completed by $4.5 \mathrm{hr}$ and further exposure to the same field did not enhance it. However, longer exposure at $50 \mathrm{~Hz}$ produced a cumulative effect on the orientation of the neurite growth. The composite line drawings shown in Figure 4 illustrate the result from one of these experiments: exposure of a culture to a pulsed field of $10 \mathrm{~Hz}$ frequency and $5 \mathrm{~V} / \mathrm{cm}$ amplitude for $24 \mathrm{hr}$ (average field intensity of $0.25 \mathrm{~V} /$

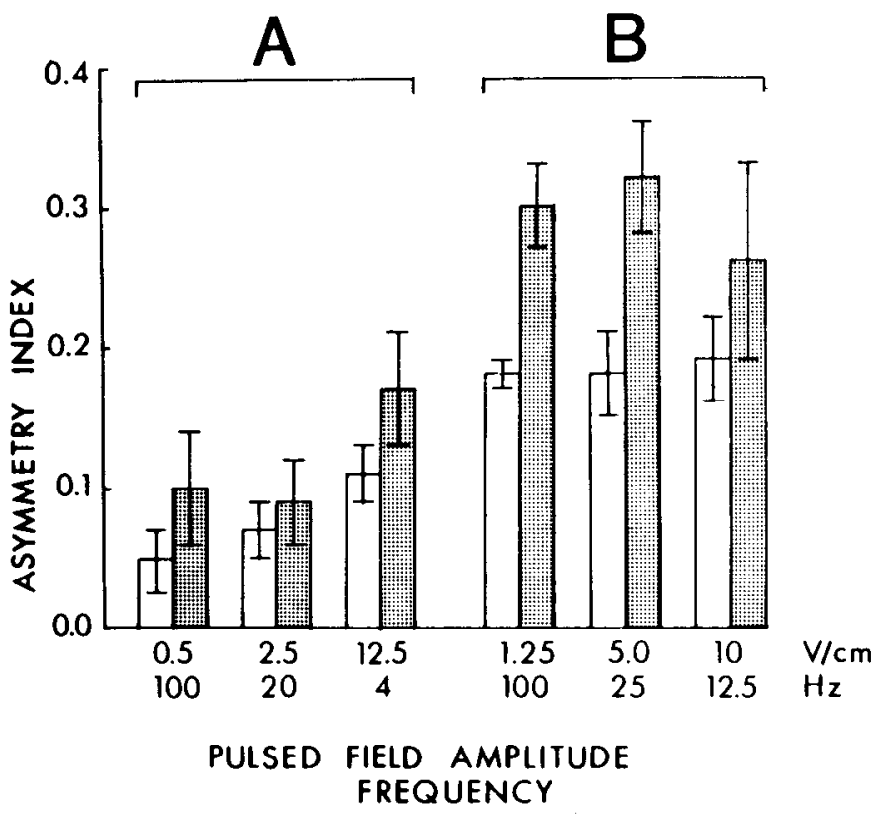

Figure 2. Asymmetry in neurite growth and neurite initiation in two groups of cultures treated with pulsed fields of different time-averaged intensities: $0.25 \mathrm{~V} / \mathrm{cm}(A)$ and $0.675 \mathrm{~V} / \mathrm{cm}(B)$. All cultures were exposed to the pulsed ficld for $4.5 \mathrm{hr}$. The time-averaged field strength was kept constant while the pulse amplitude and frequency were varied for the different cultures within each group. The pulse duration was kept constant at $5 \mathrm{msec}$. The open burs represent the neurite initiation site asymmetry (NISA) and the dotted bars represent the neurite growth asymmetry (NGA). Neurite growth and neurite initiation were significantly more asymmetric in the group exposed to the field of higher average intensity. With the exception of the case of the high pulse amplitude $(12.5 \mathrm{~V} / \mathrm{cm}, 4 \mathrm{~Hz})$, the effects produced by the fields of different combinations of pulse amplitude and frequency were similar within each group. Each bar represents the average of at least three separate cultures. Frror bars represent standard error of the mean.

$\mathrm{cm}$ ) induced curving of neurites and a higher density of neurites at the cathodal side of the cell body.

Threshold field for neurite orientation. We also examined the lowest pulsed field amplitude and frequency required to produce a detectable orientation of neurite growth over a 24-hr duration. For square pulses of $5 \mathrm{msec}$ duration, the lowest pulsed field amplitude required to produce a detectable NGA at a fixed pulse frequency of $10 \mathrm{~Hz}$ was $2.5 \mathrm{~V} / \mathrm{cm}$ (data not shown). The study shown in Figure 3 indicates that, for a fixed pulsed field amplitude of $5 \mathrm{~V} / \mathrm{cm}$, the lowest frequency required to produce a detectable neurite orientation was between 5 and $10 \mathrm{~Hz}$. Taken together, we concluded that $2.5 \mathrm{~V} / \mathrm{cm}$ and $10 \mathrm{~Hz}$ were a representative combination of the minimal pulse amplitude and frequency required to produce a detectable growth orientation in a pulsed field over a duration of $24 \mathrm{hr}$ using 5 -msec square pulses. The time-averged field intensity for this low duty cycle $(5 \%)$ field is $125 \mathrm{mV} / \mathrm{cm}$.

\section{Orientation of neurite growth by $D C$ focal field}

Steady (DC) focal fields were used to perturb the growth cone of isolated embryonic Xenopus neurons 4 to $6 \mathrm{hr}$ after plating the cells. Currents in the range 1 to $200 \mathrm{nA}$ were applied through a micropipette at a distance of $30 \mu \mathrm{m}$ from the growth cone. For each neurite, three types of observations were made: positive current, negative current, and no current, each lasting $15 \mathrm{~min}$. The sequence of these observations was set at random. The response of the growth cone was scored as + (deviation $>5^{\circ}$ toward electrode), $0\left(<5^{\circ}\right.$ deviation $)$, and - (deviation $>5^{\circ}$ 

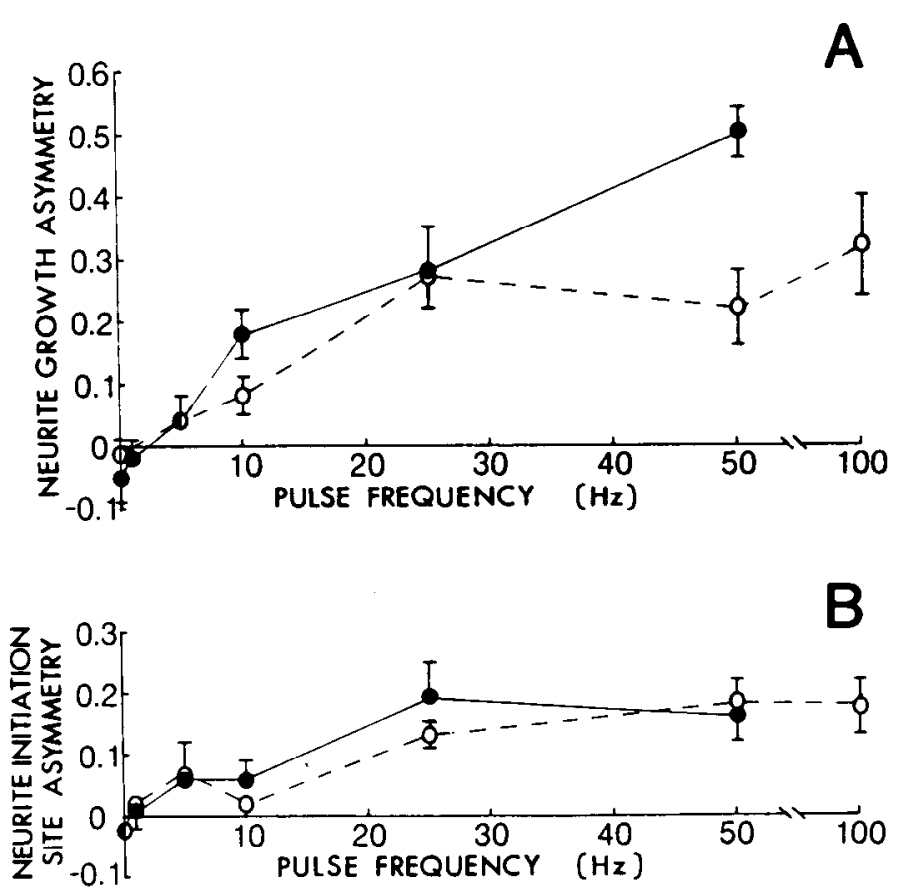

Figure 3. The frequency dependence of the pulsed field effects on the orientation of neurite growth $(A)$ and on the polarization of neurite initiation site on the cell soma $(B)$. The cultures were exposed to uniform pulsed fields of different frequencies for either $4.5 \mathrm{hr}$ (open circles) or $24 \mathrm{hr}$ (solid circles). Pulse amplitude and pulse duration were kept constant at $5 \mathrm{~V} / \mathrm{cm}$ and $5 \mathrm{msec}$, respectively. Marked neurite growth asymmetry was observed for frequencies above $10 \mathrm{~Hz}$, and above $25 \mathrm{~Hz}$ for asymmetry in neurite initiation site. Each point represents the average of at least three separate cultures. The error bars represent standard error of the mean.

away from electrode). A typical experiment is depicted in Figure 5 , which shows the responses of a growth cone to a focal current of $200 \mathrm{nA}$. In the first $15 \mathrm{~min}$ of the experiment, no current was applied; the neurite grew a length of $7 \mu \mathrm{m}$ along its original direction of growth (Fig. 5, $a$ and $b$ ). At the end of the 15-min period, the pipette was repositioned and a negative current of $200 \mathrm{nA}$ was applied. Within the subsequent $15 \mathrm{~min}$, the growth cone grew nearly $30 \mu \mathrm{m}$ toward the pipette (Fig. 5, $c$ and $d$ ). After repositioning the pipette again, a positive current of the same amplitude was applied for another $15 \mathrm{~min}$, and the growth cone deflected away from the pipette with a reduced rate of growth (Fig. 5, $e$ to g). Results from all experiments are shown in Figure 6. In the absence of any current, a fraction (38\%) of the neurites deviated $\left(>5^{\circ}\right)$ from the original direction. However, in the presence of a current there was a clear trend: many of the neurites were attracted to the current sink and deflected away from the current source within 15 min (Fig. 6). Judging from the O.I.s (see "Materials and Methods"), the threshold current strength that produced an oriented growth response above the control level was 1 and $10 \mathrm{nA}$ for the current sink and the current source, respectively. $\Lambda$ ssuming that the current spread near the vicinity of the pipette tip is radial, a 1 - to 10 nA current through the pipette yields a current density of about 0.2 to $2 \mathrm{pA} / \mu \mathrm{m}^{2}$ or a field strength of 3 to $30 \mathrm{mV} / \mathrm{cm}$ (medium resistivity $160 \mathrm{ohm}-\mathrm{cm}$ ) at the growth cone $30 \mu \mathrm{m}$ away from the pipette.

The average angle of neurite orientation for all of the neurites measured at different current strengths is shown in Figure 7. The current sink appears to be more effective in perturbing the neurite than a current source, especially at the low current levels. This asymmetry in the orientation response may be
A

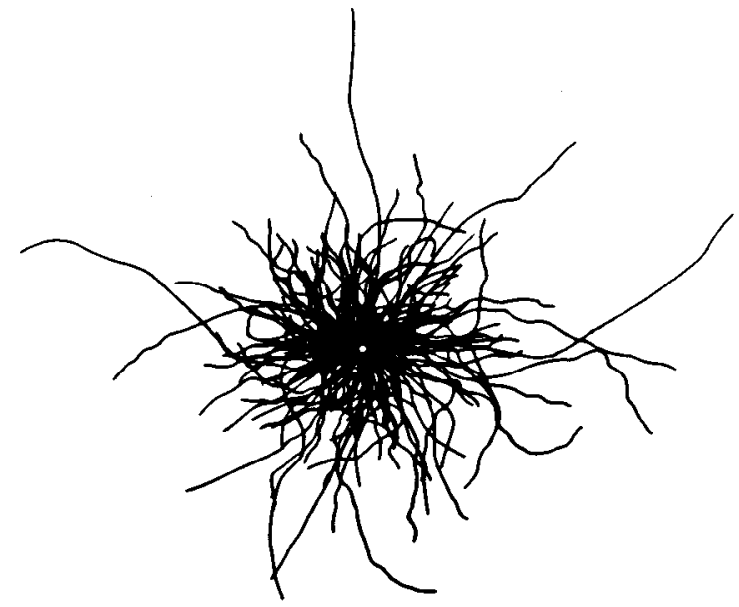

B

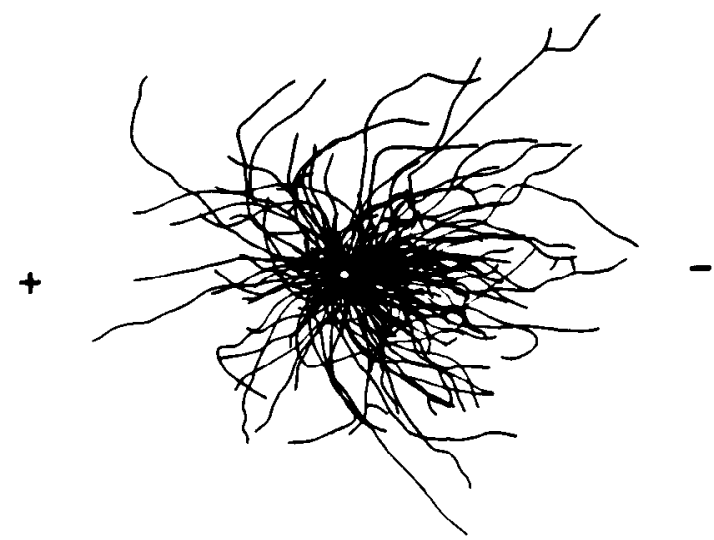

Figure 4. Composite line drawing of neurite growth from dissociated Xenopus neurons. The soma and the entire trajectory of the neurite processes of isolated neurons in the culture were traced with the aid of a camera lucida, and a composite drawing was made by superimposition upon each other of the center of all of the cell bodies of the individual neurons. $A$, Growth of 93 neurons from a 24-hr control culture (not exposed to the field). The NGA was 0.04 , and the NISA was $0.00 . B$, Growth of 110 neurons from another culture that was exposed to a pulsed field for a period of $24 \mathrm{hr}$ at a pulse frequency of $10 \mathrm{~Hz}$, pulse amplitude of $5 \mathrm{~V} / \mathrm{cm}$, and pulse duration of $5 \mathrm{msec}$. Clear neurite growth orientation toward the cathode was observed. The asymmetry indices for neurite growth orientation and for neurite initiation were 0.26 and 0.12 , respectively. $B a r=100 \mu \mathrm{m}$.

attributed in part to the fact that the deflecting growth cone will experience a progressively smaller current density as it grows away from the pipette during the course of the experiment, whereas the converse will be true for the growth toward the pipette.

\section{Orientation of neurites by pulsed focal field}

Experiments were also carried out using pulsed focal fields. Square current pulses of $5 \mathrm{msec}$ duration and different combinations of pulse amplitude and frequency were applied through a micropipette near the growth cone, in a procedure similar to that used in the focal DC field studies. Figure 8 illustrates the sequence of a typical experiment in which the response of a growth cone to $20-\mathrm{nA}$ negative current pulses at different frequencies was studied. At the onset of the experiment no current was applied; the neurite grew about $20 \mu \mathrm{m}$ along its original direction of growth in $15 \mathrm{~min}$ (Fig. 8, $a$ and $b$ ). When a current of $-20 \mathrm{nA}$ at a frequency of $10 \mathrm{~Hz}$ was applied, the neurite bent nearly $15^{\circ}$ toward the pipette within 15 min (Fig. 

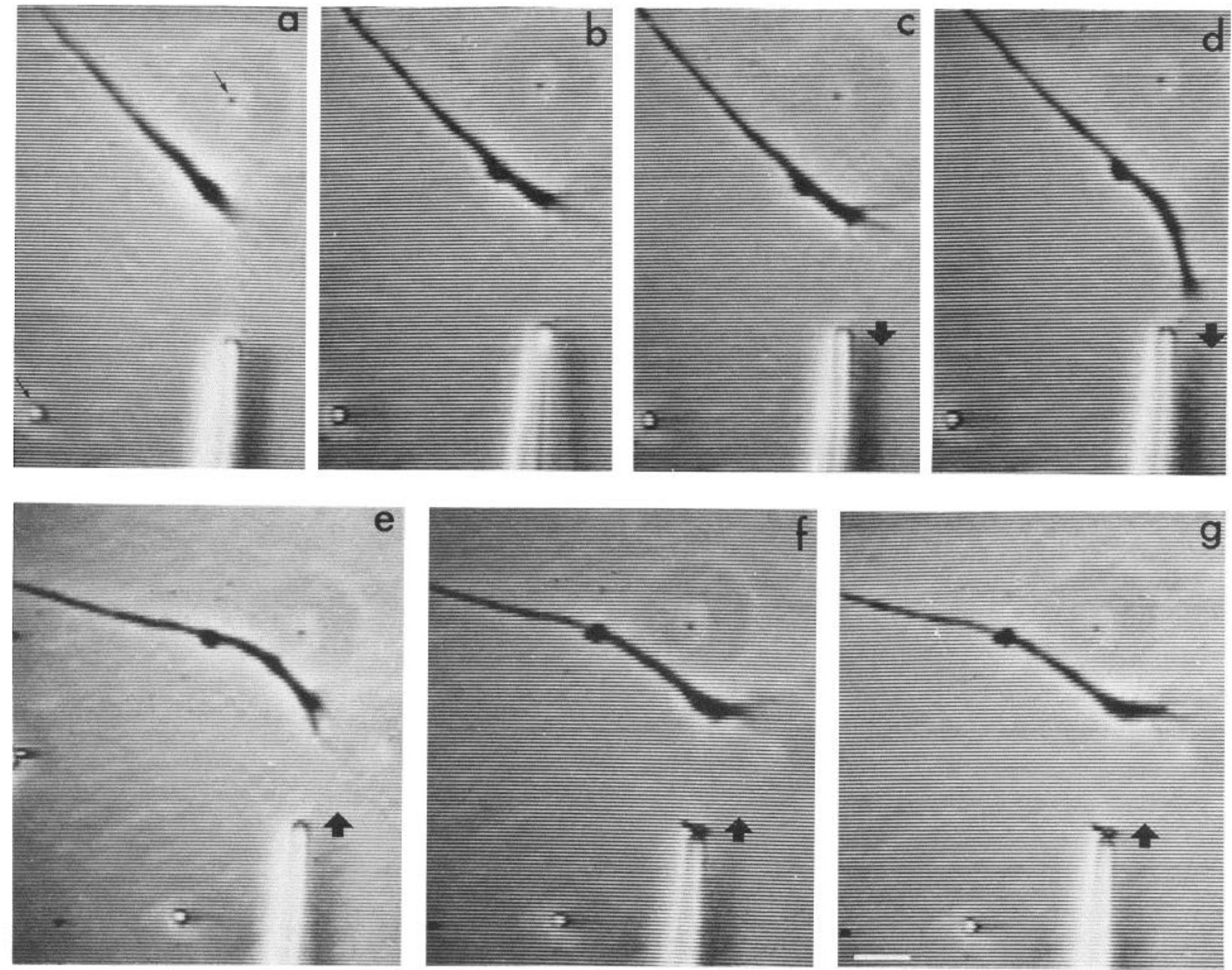

Figure 5. Bending of the growing tip of a neurite in response to a focally applied steady electric field. $a$, At the onset of the experiment, a gelfilled micropipette was placed $30 \mu \mathrm{m}$ away from the growth cone at a polar angle of $45^{\circ}$ with respect to the direction of neurite growth. $T=0$ $\min . b$, Photograph taken after $15 \mathrm{~min}$ of control growth in the absence of an electric field. $T=15$ min. $c$, At the onset of the application of a negative (inward) current of $200 \mathrm{nA} . T=20 \mathrm{~min} . d$, The oriented neurite growth recorded 15 min later. $T=35$ min. $e$, Repositioning of the pipette with respect to the growth cone and the onset of a reversed (outward) current of the same ( $200 \mathrm{nA})$ amplitude. $T=40 \mathrm{~min} . f$, The neurite grew with an apparently reduced rate during the subsequent $15 \mathrm{~min} . T=55 \mathrm{~min}$. $g$, The neurite showed a clear deflection from the pipette after $20 \mathrm{~min}$ in the reversed field. $T=60 \mathrm{~min}$. Thick arrows indicate the direction of current flow and thin arrows $(a)$ point to debris on the culture substratum that served as fixed position markers. Bar $=15 \mu \mathrm{m}$.

$8, c$ and $d$ ). The current was then turned off for $10 \mathrm{~min}$, and the neurite continued to grow in the new direction (Fig. 8e). Repositioning the pipette and applying the same current at a higher frequency $(50 \mathrm{~Hz})$ for 15 min caused the neurite to bend sharply toward the pipette (Fig. $8, e$ and $f$ ). The minimal amplitude and frequency required to induce a bending response in a neurite were variable. However, for current pulses of the same duration and amplitude, we found that the higher-frequency pulses were in general more effective. As with the DC focal fields, there was a clear trend of orientation toward the pipette when it acted as a current sink and away from the pipette when it acted as a current source. Figure 9 depicts the orientation response of single neurites to pulsed focal fields of different amplitude and frequencies, and Figure 10 shows the compiled results from all of the measurements on the effect of the pulsed focal current of negative polarity. The threshold current pulse needed to perturb the neurite at a frequency of $10 \mathrm{~Hz}$ and a pulse duration $5 \mathrm{msec}$ was about $20 \mathrm{nA}$, corresponding to an average current density of $0.2 \mathrm{pA} / \mu \mathrm{m}^{2}$ at the growth cone. The latter value was identical to that of the minimal effective DC focal current in attracting neurites (see above), suggesting again the equivalent of pulsed and focal DC fields of the same average intensity.

\section{Behavior of the growth cone in the focal field}

We have examined the filopodial activity of the growth cone under the influence of focal currents by time-lapse video recording. In many cases, response of the growth cone can be discerned immediately after the onset of the current: the number and the length of filopodial extension increased toward a current sink and decreased toward a current source, with the net result of a curved growth of the newly extended tip of the neurite. The neurite curved only when it grew; neurites that showed apparently normal undulating filopodial activity without net extension of the neurite length all failed to be oriented by the current. This observation indicates that the currentinduced orientation is likely to be associated with a preferential growth activity, e.g., formation of new filopodia, rather than a rearrangement of the pre-existing neurite. After the curving response had occurred, the distal portion of the pre-existing 


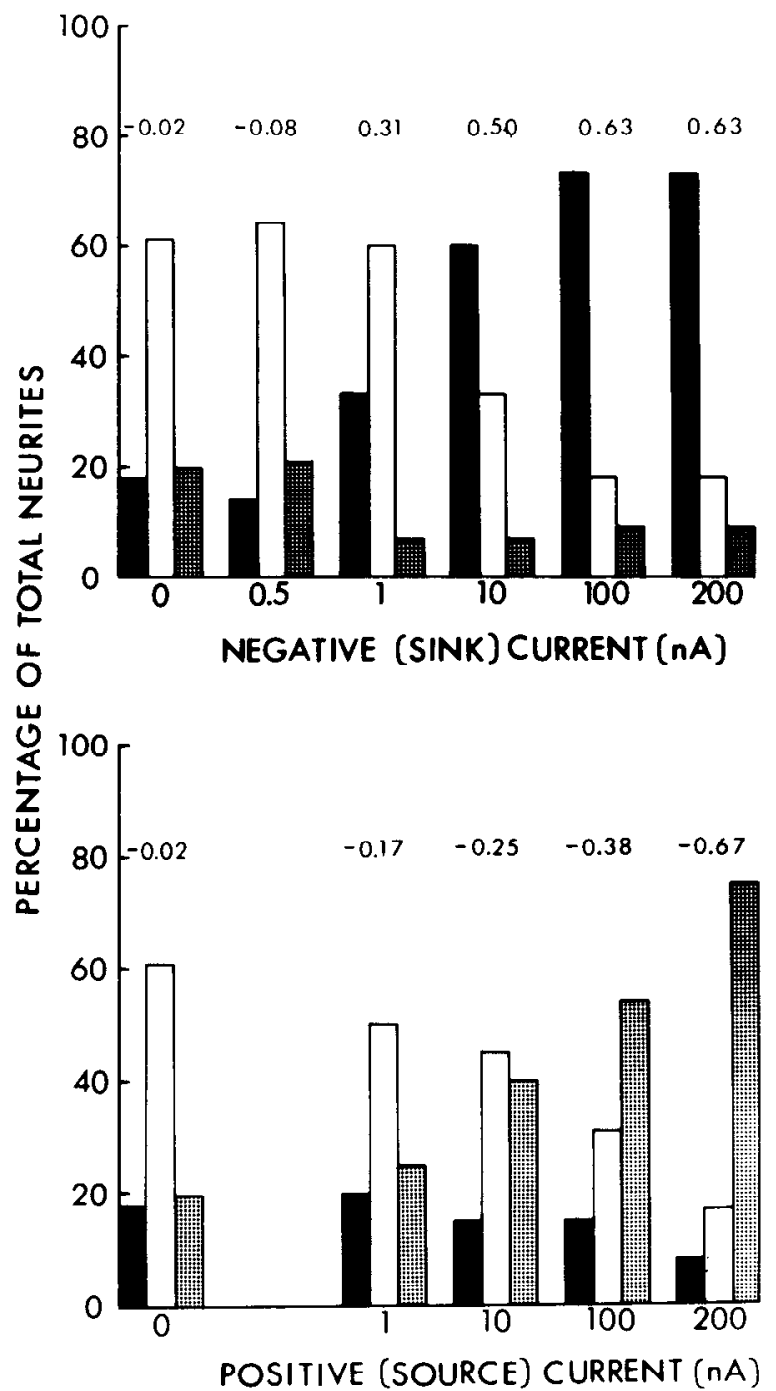

Figure 6. Effect of focally applied steady fields of different polarity and field strength on the direction of neurite growth. The fields were applied for $15 \mathrm{~min}$ in a manner similar to that illustrated in Figure 5. The histogram shows the percentage of neurites at each current strength that were attracted toward the pipette (black bars), deflected away from the pipette (dotted bars), or showed no change in the direction of neurite frequently moved to a varying degree to reduce the curvature of the neurite, presumably due to a passive response of the neurite to the tension exerted by the growth cone at its new position.

\section{Discussion}

The present study of the response of neurite growth to extracellular electric fields was conducted in two parts. In the first part, the neurites were treated with uniform pulsed fields for 4.5 and $24 \mathrm{hr}$. We observed that the neurite growth and the site of neurite outgrowth from the cell soma were preferentially oriented toward the cathodal pole of the field. These effects appear to depend directly on the time-averaged field intensity. The minimal averaged field intensity required to produce a detectable effect by a uniform pulsed field was about $125 \mathrm{mV}$ / $\mathrm{cm}$, a value within an order of magnitude to that found in previous studies using uniform DC fields (Hinkle et al., 1981; Patel and Poo, 1982; Patel, 1984). In the second part, we studied the response of the growth cones of individual neurites to focally applied electric fields of 15 min duration. We found that growth cones were attracted toward a current sink and deflected away from a current source in a manner consistent with the orientation of neurites in a uniform field. The minimal effective focal field, in terms of the time-averaged intensity, was found to be between 3 and $30 \mathrm{mV} / \mathrm{cm}$, a value 4 - to 40 -fold lower than that for the pulsed uniform fields used in the present study. However, this value was determined for the center of the growth cone. Since the actual site of the field action, whether it is at the center of the growth cone or on the extended fine filopodia (up to $15 \mu \mathrm{m}$ in length), is not certain, this value of threshold effective field may be an underestimate. Assuming a radial spread of the current, the uncertainty in the estimate of field strength can account for about 4-fold difference. Although no direct evidence is available, it is possible that the cellular

growth (white bars). The number of measurements carried out for each current strength was between 11 and 49. Negative (sink) or positive (source) currents refer to the currents flowing into or out of the focal pipette, respectively. Bars associated with 0 current were from control experiments where no current was applied through the pipette. Criteria for the bending response and the determination of the orientation indices (O.I., the numbers above the bars) are described under "Materials and Methods." The minimal current strength necessary to attract or deflect the growing tip of the neurites (O.I. $>0.2$ ) were 1 or $10 \mathrm{nA}$, respectively.

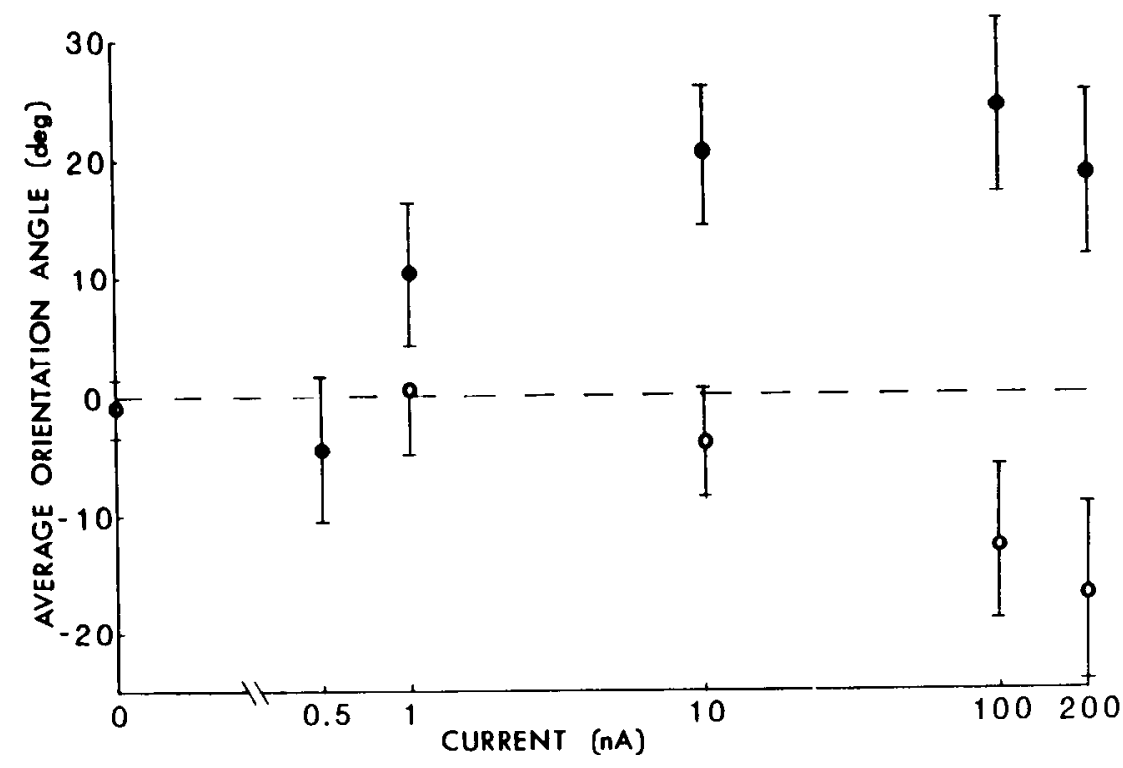

Figure 7 . The average orientation (bending) angle for all of the neurites in the presence of different focal DC fields illustrated in Figure 6. Solid circles are for fields of negative polarity (current sink) and open circles are for fields of positive polarity (current source). 'The orientation angle was measured by the procedure described under "Materials and Methods." The number of measurements for each data point is the same as that for Figure 6. Positive angles indicate bending toward the pipette, while negative angles indicate bending away from the pipette. Error bars represent standard errors. 

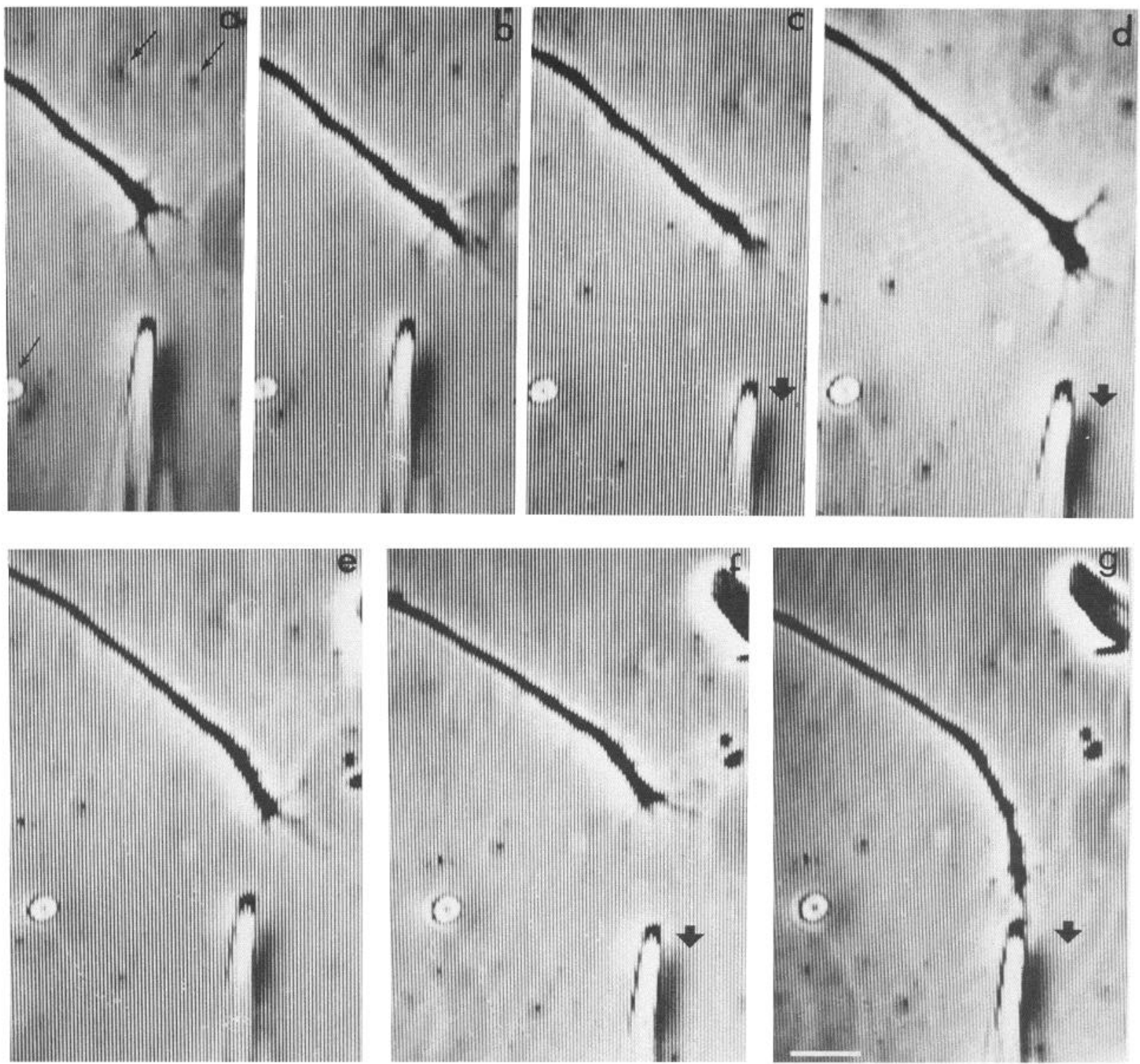

Figure 8. Orientation of the neurite growth induced by a focal pulsed field. $a$, At the onset of the experiment, $T=0$ min. $b$, Fifteen minutes of control growth in the absence of the field. $T=15 \mathrm{~min}$. $c$, After the pipette was repositioned and at the onset of negative (sink) current pulses of frequency $10 \mathrm{~Hz}$, pulse duration $5 \mathrm{msec}$, and pulse amplitude $20 \mathrm{nA}$. $T=18 \mathrm{~min}$. $d$, After $15 \mathrm{~min}$ of growth in the presence of the pulsed field, the neurite bent toward the pipette. The field was turned off at the end of the 15 -min period. $T=33$ min. $e$, Ten minutes of growth in its new direction in the absence of the field. $T=43 \mathrm{~min}$. $f$, The pipette was repositioned and the same pulsed field was reapplied with the frequency increased to $50 \mathrm{~Hz} . T=46 \mathrm{~min} . \mathrm{g}$, Fifteen minutes of growth in the second field, with further bending of the neurite toward the pipette. $T=61$ min. Thick arrows indicate the direction of the field. Thin arrows $(a)$ refer to fixed markers on the culture substratum. Bar $=15 \mu \mathrm{m}$.

machinery responsible for the turning response of the growth cone is more sensitive to perturbation by nonuniform than by uniform fields.

There is an obvious difference between the pulsed field of a certain time-averaged intensity and an equivalent steady field: for the pulsed field, any effect produced at the growth cone by each pulse is allowed to dissipate during the interpulse intervals. The finding that the effect appears to be determined by the time-averaged intensity suggests that the characteristic decay time for the cellular alteration induced by the pulsed field is much longer than the interpulse interval used in the present study; i.e., longer than $100 \mathrm{msec}$. The present study does not reveal any clue to the cellular mechanism underlying the orientation response of the neurites. Among other possibilities, an electric field may induce lateral rearrangement of neurite surface glycoproteins involved in the insertion of new membrane or the adhesion to the substratum (Poo, 1981; Patel and Poo, 1982); it may cause asymmetric alteration of membrane potential or enzyme activity on the two sides of the growth cone membrane, which in turn results in a preferential local accumulation of cytoplasmic messengers responsible for the formation of filopodia, or the incoporation of new membrane material. Whatever the mechanism, our results indicate that the cellular alteration is rapid, producing a growth response within minutes (see Fig. 8), and it is essentially additive without much dissipation for interpulse intervals on the order of $100 \mathrm{msec}$. Moreover, such cellular alterations do not persist in the absence of the field-the neurites resumed normal growth within minutes after the termination of the focal field (Fig. 8). As indicated by Figures 6 and 10, there were always some neurites that failed to respond to the focal field. Since the time required for a neurite to significantly alter its original 

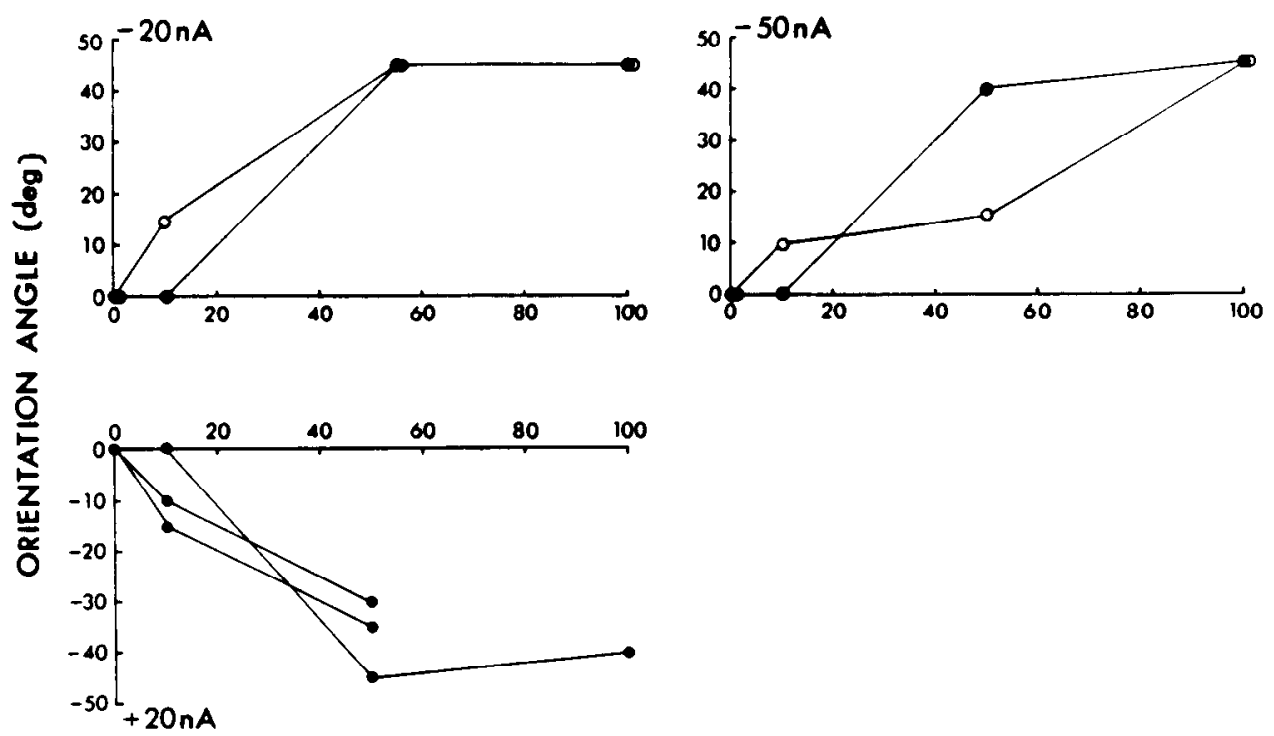

FREQUENCY $(\mathrm{Hz})$

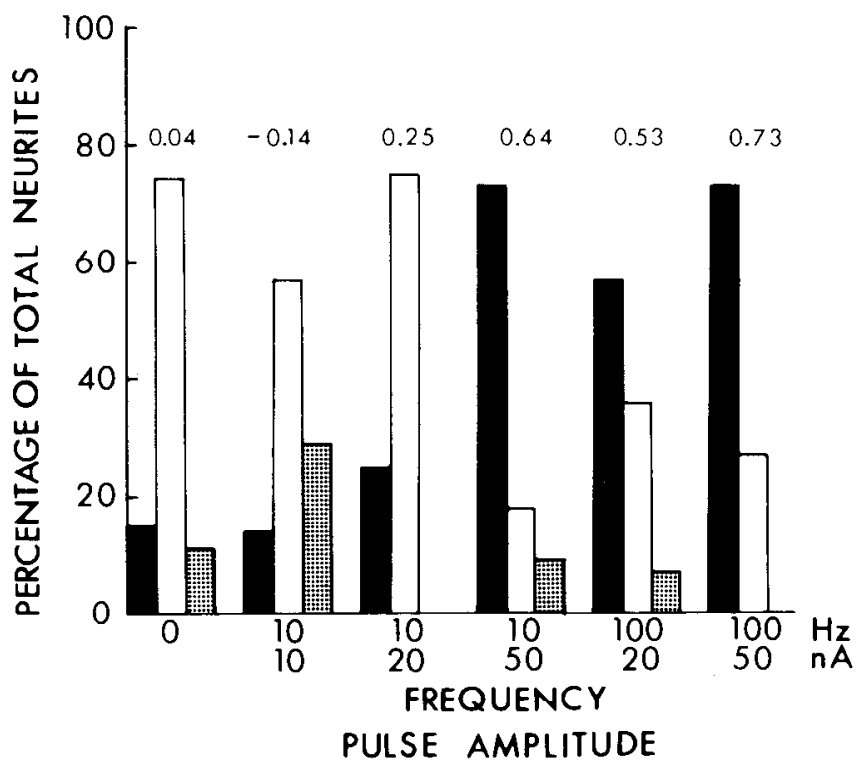

Figure 10. Attraction of the neurite growth cone by pulsed focal fields. Pulsed negative currents of various frequencies and amplitudes were applied through a focal pipette as described for Figure 8 . The pulse duration was kept constant at 5 msec. 'The histogram shows the percentage of neurites that were oriented toward the pipette (black bars) or deflected away from the pipette (dotted bars) and that showed no change from their original direction of growth (white bars). The number of measurements performed for each field was between 8 and 37. The orientation indices (O.I.s), shown above the bars, were calculated as described under "Materials and Methods." At a frequency of $10 \mathrm{~Hz}$, the amplitude of the minimal effective current was $20 \mathrm{nA}$ (i.e., O.I. $>0.2$ ).

direction of growth varies greatly, neurites that required more than $15 \mathrm{~min}$ to respond were counted by our criterion as no response. The possibility that there is a differential response of different neuronal types in our culture remains to be investigated.

The physiological significance of the electric field orientation of nerve growth has been a controversial subject (Kappers, 1917; Weiss, 1934; Jacobson, 1978; Harris, 1981). No evidence has been obtained that suggests that endogenous electric fields within the tissue serve as a guiding force for nerve growth. For in vitro systems, all types of neurons that have been studied so far responded to applied electric field. These include chick medulla and sensory ganglionic neurons (Marsh and Beams, 1946; Jaffe and Poo, 1979), Xenopus neural tube neurons (Hinkle et al., 1981; Patel and Poo, 1982), and goldfish retinal neurons (Freeman et al., 1981). The apparent lack of specificity in the field effects argues directly against the idea that the endogenous field could serve a primary role in the guidance of nerve growth. Moreover, previous studies have shown that the growing nerve can find its target cell in the absence of neuronal impulse activity (Bentley, 1975; Harris, 1980), synaptogenesis can occur even though the postsynaptic ion channels are blocked (Cohen, 1972; Giacobini et al., 1973; Obata, 1977), and regenerating nerve can find the site of the original endplate even in the absence of the muscle cell (Marshall et al., 1977). All of these results suggest that the long-range guidance of nerve growth by endogenous electric fields associated with nervous activity appears unlikely. Yet, the possibility remains that the electric field could serve a modulatory function in perturbing the neurite growth and orientation at localized regions within the nervous tissue, in a manner similar to that exhibited by the phenomena of "contact guidance" (Weiss, 1941 ) and the confinement of nerve processes in regions of high substratrum adhesiveness (Letourneau, 1975).

A net electrical current will be generated locally in the extracellular space whenever there is a segregation in the plane of membrane of ion channels and/or pumps that are responsible for the inward and outward membrane currents. This is exemplified by the specific localization of neurotransmitter receptorchannels at many synapses (Poo, 1985). It has been estimated that the peak inward synaptic current is about $2 \times 10^{-8}$ to 1.5 $\times 10^{-7} \mathrm{~A}$ at the spinal motoneurons (Eccles, 1957; Araki and Terzuolo, 1962) and about 1 to $3 \times 10^{-6} \mathrm{~A}$ at the neuromuscular junction (Fatt and Katz, 1951; Eccles and Jaeger, 1958; Takeuchi and Takeuchi, 1959). A reasonable estimate of the area of postsynaptic muscle membrane $\left(10^{3}\right.$ to $\left.10^{4} \mu \mathrm{m}^{2}\right)$ yields a current density in the range of 0.1 to $3 \mathrm{nA} / \mu \mathrm{m}^{2}$ at the synaptic cleft of a neuromuscular synapse. Estimates of synaptic current density at the spinal motoneuron also fall within this range (Ranck, 1964). Taking a rather conservative estimate of $1 \%$ as the duty cycle of synaptic current flow (e.g., for synaptic currents of duration $1 \mathrm{msec}$ and an average impulse frequency of $10 \mathrm{~Hz}$ ), the time-averaged current density experienced by the presynaptic terminal will be 1 to $30 \mathrm{pA} / \mu \mathrm{m}^{2}$. This is rather 
close to the threshold current density, namely, 0.2 to $2 \mathrm{pA} / \mu \mathrm{m}^{2}$, found to attract or deflect the growth cones in the present study. In addition to the pulsed electric currents associated with the synaptic activity, Betz et al. (1980) have also observed a steady electric current that flows out of the endplate region and re-enters the extrajunctional region of the rat skeletal muscle fiber. This steady current is of the opposite polarity to that of the synaptic current and is about one to two orders of magnitude smaller than the effective current found in the present study in orienting neurite growth.

Based on theoretical grounds, a possible direct electrokinetic effect of synaptic currents on the structure and function of synapse has been suggested previously (Ranck, 1964; Elul, 1966). The present study provided direct demonstration that focal currents of a strength similar to that of the synaptic current could perturb the growth and orientation of a nerve terminal. In addition, we have shown that the focal field provides a simple means of perturbing the nerve growth cone that may prove to be useful for studying the growth and orientation of nerve processes.

\section{References}

Araki, T., and C. A. Terzuolo (1962) Membrane currents in spinal motorneurons associated with the action potential and synaptic activity. J. Neurophysiol. 25: 772-789.

Bentley, D. (1975) Single gene cricket mutations: Effects on behavior, sensory neurons and identified interneurons. Science 187: 760-764.

Betz, W. J., J. H. Caldwell, R. R. Ribchester, K. R. Robinson, and R. F. Stump (1980) Endogenous electric field around muscle fibres depends on the $\mathrm{Na}^{+}-\mathrm{K}^{+}$pump. Nature 287: 235-237.

Cohen, M. W. (1972) The development of neuromuscular connections in the presence of D-tubocurarine. Brain Res. 41: 457-463.

Eccles, J. C. (1957) The Physiology of Nerve Cells, Johns Hopkins University Press, Baltimorc.

Eccles, J. C., and J. C. Jaeger (1958) The relationship between the mode of operation and the dimensions of the junctional regions at synapses and motor end-organs. Proc. R. Soc. Lond. Biol. 148: 3856.

Elul, R. (1966) Dependence of synaptic transmission on protein metabolism of nerve cells: A possible electrokinetic mechanism of learning? Nature 210: 1127-1131.

Fatt, P., and B. Katz (1951) An analysis of the end-plate potential recorded with an intracellular electrode. J. Physiol. (Lond.) 115: 320370.

Freeman, J. A., J. M. Weiss, G. J. Snipes, B. Mayes, and J. J. Norden (1981) Growth cones of retinal neurites generate DC currents and orient in an electric field. Soc. Neurosci. Abstr. 7: 550.

Giacobini, G., G. Filogamo, M. Weber, P. Boquet, and J. -P. Changeux
(1973) Effects of a snake $\alpha$-neurotoxin on the development of innervated skeletal muscles in chick embryo. Proc. Natl. Acad. Sci. U. S. A. $70: 1708-1712$.

Harris, W. A. (1980) The effect of eliminating impulse activity on the development of the retino-tectal projection in salamanders. J. Comp. Neurol. 194: 303-317.

Harris, W. A. (1981) Neural activity and development. Annu. Rev. Physiol. 43: 689-710.

Hinkle, L., C. D. McCaig, and K. R. Robinson (1981) The direction of growth of differentiating neurons and myoblasts from frog embryos in an applied electric field. J. Physiol. (Lond.) 314: 121-135.

Jacobson, M. (1978) Developmental Neurabiology, Ed. 2, pp. 160-166, Plenum Press, New York.

Jaffe, L. F., and M-m. Poo (1979) Neurites grow faster towards the cathode than the anode in a steady field. J. Exp. Zool. 209: 115-128.

Kappers, C. U. A. (1917) Further contributions on neurobiotaxis. IX. An attempt to compare the phenomena of neurobiotaxis with other phenomena of taxis and tropism. J. Comp. Neurol. 27: 261-298.

Letourneau, P. C. (1975) Cell-to-substratum adhesion and guidance of axonal elongation. Dev. Biol. 44: 92-101.

Marsh, G., and H. W. Beams (1946) In vitro control of growing chick nerve fibers by applied electric currents. J. Cell. Comp. Physiol. 27: 139-157.

Marshall, L. M., J. R. Sanes, and U. J. McMahan (1977) Reinnervation of original synaptic sites on muscle fiber basement membrane after disruption of the muscle cells. Proc. Natl. Acad. Sci. U. S. A. 74: 3073-3078.

Nieuwkoop, P. O, and J. Faber (1962). Normal Table of Xenopus laevis, Elsevier-North Holland Publishing Co., Amsterdam.

Obata, K. (1977) Development of neuromuscular transmission in culture with a variety of neurons and in the presence of cholinergic substances and TTX. Brain Res. 119: 141-153.

Patel, N. B. (1981) Perturbation of neurite growth in vitro by extracellular electric fields. Ph.D. dissertation, University of California, Irvine.

Patel, N. B., and M-m. Poo (1982) Orientation of neurite growth by extracellular electric fields. J. Neurosci. 2: 483-496.

Poo, M-m. (1981) In situ electrophoresis of membrane components. Annu. Rev. Biophys. Bioeng. 10:245-276.

Poo, M-m. (1985) Mobility and localization of proteins in excitable membrane. Annu. Rev. Neurosci., in press.

Ranck, J. B. (1964) Synaptic "learning" due to electroosmosis: A theory. Science 144: 187-189.

Sisken, B. F., and S. D. Smith (1975) The effects of minute directed electrical currents on cultured chick embryo trigeminal ganglia. J. Embryol. Exp. Morphol. 33: 29-41.

Takeuchi, A., and N. Takeuchi (1959) Active phase of frog's end-plate potential. J. Neurophysiol. 22: 395-411.

Weiss, P. (1934) In vitro experiments on the factors determining the course of the outgrowing nerve fiber. J. Exp. Zool. 68: 393-448.

Weiss, P. (1941) Nerve patterns: The mechanics of nerve growth. Third Growth Symp. 5: 163-203. 